\title{
Bioanalysis
}

\section{7th Japan Bioanalysis Forum symposium: regulated bioanalysis, to a new stage}

\section{Tower Hall Funabori, Tokyo, Japan, 9-10 March 2016}

At the 7th Japan Bioanalysis Forum symposium, bioanalytical methods and processes were discussed with authorities after the issuing of bioanalytical method validation guidelines (EMA and Japanese Ministry of Health, Labour and Welfare) and draft guidance (US FDA). Method establishment and bioanalysis of biomarkers were discussed with a focus on scientific validation. Bioanalytical methods for antibody-drug conjugates, the potential of MS imaging and microsampling activity in drug development were introduced. Discussion groups presented and openly discussed their results with about 300 attendees. This manuscript provides an overview of the highlights of the symposium.

First draft submitted: 4 July 2016; Accepted for publication: 31 August 2016;

Published online: 14 September 2016

Keywords: ADC • bioanalysis $\bullet$ biomarker $\bullet$ DG $\bullet$ guidance $\bullet$ guideline $\bullet$ JBF $\bullet$ symposium

A few years have passed since the guidelines and draft guidance for bioanalytical method validation (BMV) were issued [1-4], and BMV methods and processes have since changed. The Japan Bioanalysis Forum (JBF) [5] carried out a survey on this topic and discussions were held with authorities in Japan, the USA and the EU. The audit perspective for bioanalysis assay may also have changed following the issuance of BMV guidelines/guidance, and this was surveyed by the Japan Society of Quality Assurance (JSQA). The European Bioanalysis Forum (EBF) published a White Paper on method establishment and bioanalysis of biomarkers in 2012 [6]. The JBF biomarker task force attempted discussion for the preparation of the concept paper on BMV in 2015 [7], and BMV for biomarkers was discussed in the Crystal City VI workshop (CC-VI) in 2015 [8]. The panel discussion on biomarker assay was held against the background on the basis of these events. Recently, the development of antibody-drug conjugates (ADCs) has increased, and the complex bioanalysis methods associated with them were introduced. MS imaging (MSI) is a new technique for drug development, and its current status and perspectives on its use were discussed. Microsampling is one of the issues under discussion at the International Council on Harmonization (ICH) S3A and the concept paper was published by the $\mathrm{ICH}$ steering committee [9]. The schedule for the ICH S3A Questions and Answers (Q\&A) was introduced, and the survey results were presented. Seven discussion groups, in which bioanalytical issues relating to their topics of interest were discussed, presented the outcomes of their discussions. They and attendees openly exchanged their views and thoughts.

About 300 people attended this symposium and discussed bioanalytical issues. The detailed presentation materials can be found on the JBF website [5].
Takahiro Nakamura

Shin Nippon Biomedical Laboratories Ltd., 2438, Miyanoura, Kagoshima, 891-1394, Japan

Tel.: +81992942600

Fax: +81992943619

nakamura-takahiro@snbl.co.jp 


\section{Open discussion with regulatory authorities}

JBF carried out a survey among Japanese pharmaceutical companies and contract research organizations regarding bioanalysis and the BMV guidelines (EMA and MHLW) and draft guidance (US FDA).

In Part 1 (Q\&A session), Daisuke Iwata (Pharmaceuticals and Medical Devices Agency [PMDA], Tokyo, Japan), Toru Yamaguchi (PMDA) and Brian Booth (Office of Clinical Pharmacology, FDA, MD, USA) participated as panelists and expressed their personal views, and the preanswered comments from Jan Welink (EMA, Utrecht, The Netherlands) were also presented. In Part 2 (Q\&A session, ensuring reliability in drug applications), Yoshinobu Yamai (JSQA; Taisho Pharmaceutical, Tokyo, Japan) joined the discussion as a panelist.

Part 1:

- Incurred sample reanalysis (ISR): The necessity of ISR was debated when quantification results in most samples are lower than the low QC concentration. The calibration range should be set for the evaluation of PKs;

- Bioequivalence (BE) studies: There was debate regarding the acceptability of reanalysis after obtaining abnormal PKs results. If the cause is an unambiguous analytical failure, it may be reasonable to reanalyze; however, some issues deemed as bioanalytical error may be subjective, and reanalysis in these cases is not acceptable;

- Stability: If the stability shows concentration dependency, it is reasonable to assess stability at concentrations higher than the ULOQ. It may be necessary to assess stability in whole blood on a case-by-case basis, depending on the structure of the analytes;

- Tiered approach: The employees responsible for this should consult with stakeholders (pharmacology and/or toxicology groups) in the therapeutic review divisions concerning these proposals;

- Biopharmaceutical and LBA: It was confirmed that the stability and quality of critical reagents can be generally verified by monitoring the results of QCs in daily analysis;

- Validation: If cross-validation cannot be conducted, a suitable approach would be to make comparisons between PK study results and PK analysis performed by different laboratories as covariates. A partial validation for an analyst change may need to be considered on a case-by-case basis, if the bioanalytical method uses specific, nonstandard procedures and depending on the training of the analysts at the laboratory;

- Future perspective: New guidance/guidelines depend on the number of applications submitted and the difficulties associated with these applications.

Part 2:

- Changes before and after issuance of the BMV guidelines: The most specific change is the request for ISR;

- Study records: It was confirmed that, based on the condition and maintenance of analytical instruments and performance of system suitability, the change to the same or similar type of original instrument is reasonable for recording raw data without partial validation;

- Self-check: It depends on the each drug company's approach whether QC is performed for all data or whether data are extracted from the common technical document and original reports;

- Blindness: Blindness should be maintained even in biomarker assays;

- Validation reports: It is acceptable to start sample analysis before finalizing a method validation report, if major validation experiments are completed;

- Generic drugs: The purpose of ISR is to confirm the repeatability of PK study end points for BE studies; therefore, ISR should be performed even in preliminary BE studies.

\section{Audit perspective for bioanalysis assay}

JSQA is considering quality assurance for medicines and technical proposals, and the KT-2 subgroup of the Common Special Project Group 2 in JSQA is examining QA methodology at clinical/medical laboratories for clinical trials. They conducted a questionnaire on the implementation of a bioanalysis in clinical trials' targeted at the JSQA members. Tomoyuki Tabata (JSQA; Ajinomoto Pharmaceutical, Tokyo, Japan) and Yoshinobu Yamai presented the survey results in the poster session, and Yoshinobu Yamai also presented them in the oral session.

The KT-2 members were interested in ISR and reanalysis. They highlighted the survey results about these items. Auditors made some points regarding bioanalysis assays. Auditors thought that it was sufficient to carry out ISR in bioanalysis assays after the issuance of BMV guidelines/guidance. They showed 
much interest in reanalysis, confirmed that reanalysis were conducted with the data adopted according to standard operating procedures and/or protocols and ensured that these procedures were described in the reports as reanalyses. In the $\mathrm{BE}$ study, it was difficult to permit either the adoption or rejection of reanalysis results, conducted without definite descriptions in standard operating procedures and/or protocols. They also presented the other survey results.

\section{Panel discussion of biomarker assay}

At first, Philip Timmerman (EBF; Janssen R\&D) and Marianne Scheel-Fjording (EBF; Novo Nordisk, Maaløv, Denmark) explained EBF's activities for bioanalytical method development and validation in biomarker assays. The EBF published a White Paper for method establishment and bioanalysis of biomarkers in support of drug development in 2012 [6]. EBF has recommended making decision about biomarker assay requirements by considering the following four classifications:

- Observed or anticipated biomarker level changes

- Development phase in which a biomarker is measured

- Decisions taken from the biomarker data

- Possible fit of the assay specifics with regulated bioanalysis guideline

The EBF conducted a survey of 57 statements in 2015. From this survey EBF took home the following five messages:

- Generic criteria are difficult for all biomarker assays

- Discussion should include all stakeholders

- Assay requirements can change

- Global consensus should be sought

- Principles of scientific validation should be used

After this survey, CC-VI was held at Baltimore in 2015 [8]. The messages from CC-VI were very similar to the outcome of EBF's survey, and the FDA expressed that they are in learning mode, and do not expect guidance for several years to come.

In the panel discussion, Kousuke Iijima (Kyowa Hakko Kirin, Shizuoka, Japan), Toshihiro Oguma (Daiichi Sankyo, Tokyo, Japan), Yoshiaki Ohtsu (JBF; Astellas Pharma, Ibaraki, Japan) and Takashi Miyayama (Chugai Pharmaceutical, Kanagawa, Japan) joined as panelists, and focused on a practical approach for method validation of biomarker assays based on the concept of scientific validation. In the EBF community, most companies ( $\sim 75 \%)$ have already introduced, or are considering the idea of scientific validation. In Japan, some companies already introduced this validation concept several years ago. A key aspect of scientific validation is proper communication between bioanalysts and stakeholders of biomarker assays, including biologists. The mutual recognition of assay reliability is especially essential for the successful biomarker assay.

\section{Round table talks on biomarker assays}

Though biomarkers have been expected to heavily contribute to the discovery and development of new drugs, successful case studies are very limited with the exception of genomic biomarkers. Noboru Nakayama (Biosys Technologies, Tokyo, Japan) presented on recent activity for multiple instances of biomarker research in cancerous tissues. The components of several molecules changed according to the stage of the cancer. Interestingly, the change of components was also detected in the precancerous stage. These findings suggest that tracing multiple molecules might bring about a new breakthrough in biomarker assays for new drug discovery.

\section{Antibody-drug conjugates}

Rand Jenkins (Pharmaceutical Product Development, VA, USA) covered the broad topics of bioanalysis of ADCs from the basic methods to the latest applications. After the launch of Kadcyla and Adcetris, many ADCs are being developed. LBAs have traditionally been used for ADC bioanalysis as a gold standard to determine total monoclonal antibody and conjugated monoclonal antibody concentrations. However, the presence of soluble target in the sample and specificity of critical reagents often affect the assay responses. The alternative LC-MS-based methods that employ the affinity capture technique are now being applied to measure various ADC species, especially for nonclinical assays. ADCs are captured from plasma or serum samples using anti-human Fc antibody or Protein A/G and the bound proteins are measured by LC-MS/MS after trypsin digestion.

In the latter part of his presentation, he mentioned the advantages and challenges offered by affinity capture LC-MS. The characterization of drug-antibody ratio is useful to assess the stability and biotransformation of ADCs and specificity of critical reagents for LBAs. LC-MS approach also can be used to evaluate the change of interchain disulfide and site-specific catabolism of the drug while still conjugated to the antibody. In addition, the recent progress of highresolution MS coupled with affinity capture allows direct measurement of intact ADC changes with good 
accuracy and precision. He concluded that both LBA and LC-MS assays are needed to keep up with innovation in biotherapeutics and MS-based methods are emerging as increasingly versatile tools.

\section{Current status \& perspective on MSI in drug development}

At first, Mitsuhiro Hayashi (National Cancer Center, Tokyo, Japan) introduced the basis of MSI, and presented drug distribution in nonclinical studies and translational research using MSI in cancer research. Yuuya Houjyou (Japan Pharmaceutical Manufacturers Association [JPMA]; Kyorin Pharmaceutical, Tokyo, Japan) introduced JPMA activities for MSI. Evaluation of efficacy and toxicity based on information on the drug and its metabolite distribution in tissues, and simultaneous analysis of drugs, biomarkers and metabolomics in the same specimens are expected. But there are issues in the MSI technique and utilization of the data thus obtained. The cooperation of the industry, academia and government is important, and upgrades of technique and standardization of MSI procedures are needed. Shintaro Nakano (PMDA) presented on the current conditions of drug distribution studies in the approval application documents, the expectation to leverage MSI for drug development in studies, and the acquisition of useful information for drug efficacy and toxicity. In the panel discussion, it was noted that reliability assurance is important for leveraging MSI; and that the use of MSI as a substitute for quantitative whole-body autoradiography is controversial because MSI is not as widely used as LC-MS at this time.

\section{Microsampling: ICH S3A Q\&A \& panel discussion}

First, Noriko Katori (Deputy Topic Leader of ICH S3A Q\&A, NIHS) presented the ICH S3A Q\&A update. After reading information about the $\mathrm{ICH}$ association and ICH process for new guidelines, she explained the background, purpose, progress, discussion points and schedule for the ICH S3A Q\&A. Second, Kozo Omichi (DG17 [microsampling]; Kowa, Shizuoka, Japan) presented the survey results regarding the use of microsampling in the Japanese pharmaceutical industry and the issues involved in its use. Considering these present conditions, a panel discussion commenced. Three EWG members of ICH S3A Q\&A, Yoshiro Saito (Rapporteur, Topic Leader and Regulatory Chair, NIHS), Shinichi Sekizawa (PMDA) and Kazuhiro Taniyama (JPMA; Toa Eiyo, Fukushima, Japan) and DG17 reader, Eitaro Nanba joined as panelists and Noriko Katori and Keiko Nakai (LSI Medience, Tokyo, Japan) facilitated the panel discussion. Expectations concerning microsampling, the use of satellite groups, the priority of microsampling relative to ISR, clinical microsampling approaches and technical difficulty were all discussed.

\section{Discussion group}

Yoshihisa Sano (JBF; Sunplanet, Ibaraki, Japan) organized this session. The leaders of seven discussion group (DGs) (DG13 to 19) introduced their activities briefly in the oral session (on Day 1) and the members of each DG presented their outcomes and openly exchanged their views on a wide range of bioanalytical issues with symposium attendees in the poster session (on Day 2).

DG13 discussed the topic of 'Questions and challenges in bioanalytical study - find the loadstar,' focusing on small-molecule quantification. The group consisted of 12 members (leader: Naohito Yamada; Japan Tobacco, Kanagawa, Japan). The items discussed include calibration curves, ISR, data assessment and others (e.g., quantification range using narrow calibration curve, ISR failure and definition of chromatogram reintegration) based on questions collected from DG supporters, and they also shared proposed solutions and recommendations in response to questions. Additionally, they conducted a survey at the symposium venue to share the current practice in Japan and to refine their discussion.

DG14 discussed the topic of 'Carryover.' The group consisted of six members (Leader: Takumi Noda; Ono Pharmaceutical, Fukui, Japan). DG14 surveyed experiences of the carryovers that DG supporters have faced, and spent much time debating among the members. Four topics about carryovers were selected and reported: first, the identification of the root of the carryover; second, the measures taken to minimize and/or eliminate the carryover in each scenario; third, the actual examination items and time periods for the carryover solutions; and finally, the strategies of unknown sample analysis using a method with unavoidable carryovers in PK/TK studies.

DG15 discussed the topic of 'Quantitative analysis of endogenous substances (2),' following the discussion held as DG08 in 2015, that focused on the selection of an alternative matrix for quantitative assays of endogenous substances in biological samples. The group consisted of 17 members with different backgrounds who use LC-MS and LBA in their daily tasks (Leader: Kazuaki Sakai; Teijin Pharma, Tokyo, Japan). They proposed:

- A strategy for selecting the appropriate alternative matrix

- How to verify the selected alternative matrix, for the quantitative assays from the different stand points of LC-MS and LBA 
DG16 discussed the topic 'Scientific validation.' The group consisted of 11 members (leader: Makoto Niwa; Nippon Kayaku, Tokyo, Japan). They shared their interpretation of the recommendation by EBF [10] and considerations for the characterization of items for scientific validation. The scientific validation items discussed were metabolites in relation to ICH-M3(R2), urine analysis, tissue (homogenate) analysis, early stage clinical studies and early development preclinical studies. The DG plans to work on additional scientific validation items, for example, in vitro studies.

DG17 focused on the topic of 'Microsampling.' The group consisted of nine members (leader: Eitaro Nanba; Chugai Pharmaceutical, Shizuoka, Japan). They considered that it was necessary to clarify the issues involved in the application of microsampling and to discuss solutions among members of the Japanese drug industry in order to promptly introduce and promote the use of the microsampling. They conducted a survey regarding how to define microsampling, how it is currently implemented in Japan and what issues it faces. The survey results were also presented in the microsampling session on the second afternoon.

DG18 discussed the topic of 'analysis of anti-drug antibody (ADA).' The group consisted of nine members (leader: Tatsuki Nomura; Shin Nippon Biomedical Laboratories, Kagoshima, Japan,). They discussed ADA analysis of patients, validation of neutralizing antibody assay, no positive reaction in clinical phase, verification of acid dissociation, critical reagents, trouble shooting, strategy of ADA analysis and necessity/achievement of neutralization activity and isotypes, and finally expressed their consideration to propose a standard procedure for the ADA assay.

DG19 focused on the topic of 'Quantitative analysis by LBA (Method development).' The group consisted of six members (leader: Hiroyuki Shimizu; Toray Research Center, Kanagawa, Japan). The members discussed the development of a method including experimental procedures for three LBA platforms (MSD, Gyrolab and ELISA), the selection of a cali-

\section{References}

1 EMA. Guideline On Bioanalytical Method Validation. European Medicines Agency, London, UK (2011).

www.ema.europa.eu

2 Japanese Ministry of Health, Labour and Welfare. Guideline on Bioanalytical Method Validation in Pharmaceutical

Development. Japanese Ministry of Health, Labour and Welfare, Tokyo, Japan (2013).

3 US Department of Health and Human Services, FDA. Draft Guidance for Industry: Bioanalytical Method Validation. US FDA, USA (2013). www.fda.gov bration standard for biomarker analysis, the measurement of drug concentration in tissue, the training of laboratory scientists and the verification of the data equivalence among scientists, to achieve efficient LBA analysis.

\section{Conclusion}

BMV methods and processes were standardized after the issuance of BMV guidelines/guidance. Biomaker bioanalysis was discussed with a focus on scientific validation. New technologies (ADCs, MSI and microsampling) might change current concepts in drug development. We have to contribute to delivering safe and effective pharmaceuticals quickly to patients by facilitating scientific discussions on bioanalysis among scientists, bioanalytical communities and regulatory agencies.

JBF has continuously been involved as the sole form for regulated bioanalysis in Japan, and JBF plans to hold the 8th JBF symposium in Tokyo, Japan on 8-9 February 2017.

\section{Acknowledgements}

As the host of the symposium, the author would like to thank: A Nakayama (Ajinomoto, Kanagawa, Japan) and S Tanaka (JPMA; Aska Pharmaceutical, Kanagawa, Japan) for their fruitful sessions, all speakers and panelists for their meaningful presentations and opinions, the members of the JBF steering committees for their dedication to this symposium organization, and JBF DG members and symposium attendees for their considerable discussion.

\section{Financial \& competing interests disclosure}

The author has no relevant affiliations or financial involvement with any organization or entity with a financial interest in or financial conflict with the subject matter or materials discussed in the manuscript. This includes employment, consultancies, honoraria, stock ownership or options, expert testimony, grants or patents received or pending, or royalties.

No writing assistance was utilized in the production of this manuscript.

4 Japanese Ministry of Health, Labour and Welfare. Guideline on Bioanalytical Method (Ligand Binding Assay) Validation in Pharmaceutical Development. Japanese Ministry of Health, Labour and Welfare, Tokyo, Japan (2014).

5 Japan Bioanalysis Forum. http://bioanalysisforum.jp/en

6 Timmerman P, Herling C, Stoellner D et al. European Bioanalysis Forum recommendation on method establishment and bioanalysis of biomarkers in support of drug development. Bioanalysis 4(15), 1883-1894 (2012).

7 Igarashi H. 6th Japan Bioanalysis Forum symposium: challenge of regulated bioanalysis. Bioanalysis 7(14), 1711-1715 (2015). 
8 Lowers S, Ackermann B. AAPS and US FDA Crystal City VI workshop on bioanalytical method validation for biomarkers. Bioanalysis 8(3), 163-167 (2016).

9 Final Concept Paper, S3A: Q\&As on Note for Guidance on Toxicokinetics: the Assessment of Systemic Exposure, Focus on Microsampling. ICH Steering Committee (2014).

www.ich.org
10 Timmerman P, White S, McDougall S et al. Tiered approach into practice: scientific validation for chromatography-based assays in early development - a recommendation from the European Bioanalysis Forum. Bioanalysis 7(18), 2387-2398 (2015). 